
\title{
28 Research Square \\ Label-free enrichment of rare unconventional circulating neoplastic cells using a microfluidic dielectrophoretic sorting device
}

Jose Montoya Mira

Oregon Health \& Science University

Ajay Sapre

University of California, San Diego

Brett Walker

Oregon Health \& Science University

Jesus Bueno Alvarez

Oregon Health \& Science University

Kyle Gustafson

Oregon Health \& Science University

\section{Eugene Tu}

Oregon Health \& Science University

Jared Fischer

Oregon Health and Science University https://orcid.org/0000-0002-0062-0870

Melissa Wong

Oregon Health \& Science University

\section{Sadik Esener}

Oregon Health \& Science University https://orcid.org/0000-0002-6873-0478

Yu-Jui Chiu ( $\square$ chiuyu@ohsu.edu )

Oregon Health \& Science University

\section{Article}

Keywords: Cancer, dielectrophoresis, circulating hybrid cell, liquid biopsy, KRAS, circulating tumor cell

Posted Date: May 10th, 2021

DOI: https://doi.org/10.21203/rs.3.rs-415667/v1

License: (c) (1) This work is licensed under a Creative Commons Attribution 4.0 International License.

Read Full License 
Version of Record: A version of this preprint was published at Communications Biology on September 24th, 2021. See the published version at https://doi.org/10.1038/s42003-021-02651-8. 


\section{Abstract}

Technologies that facilitate analyses of circulating biomarkers from blood for cancer detection are powerful tools for improving patient outcomes. Circulating biomarkers derived directly from the primary tumor have been identified including circulating tumor cells (CTCs) and circulating hybrid cells (CHCs), described to harbor phenotypes of both tumor cells and leukocytes. CHCs are present in higher numbers than CTCs which support their translational potential. Methods for isolation of $\mathrm{CHCs}$ do not exist and are restricted to low-throughput, time consuming, and biased methodologies. Here, we report development of a label-free dielectrophoretic (DEP) microfluidic platform facilitating enrichment of $\mathrm{CHC}$ in a highthroughput and rapid fashion. Unlike other methods, depletion of healthy peripheral blood mononuclear cells (PBMCs) drives enrichment of CHCs. We analyzed DEP differential response of PBMCs and cancer cells and demonstrated up to $96.5 \%$ depletion of PBMCs resulting in 18.6-fold enrichment of cancer cells. In PBMCs from pancreatic adenocarcinoma patients, the platform enriched for neoplastic cells identified by their KRAS mutant status using droplet digital PCR from only $2 \mathrm{~mL}$ of whole blood with one hour of processing. Enrichment was achieved in $75 \%$ of the clinical samples analyzed, establishing this approach as a promising way to non-invasively analyze tumor cells from patients.

\section{Introduction}

Cancer is a major worldwide public health burden and the second leading cause of death in the United States [1]. Advances in medical technologies led to significant progress in both treatment and diagnosis, yet clinicians rely on invasive and often risky biopsies for cancer detection. To address these limitations, the field of liquid biopsy has rapidly emerged as a non-invasive method to obtain tumor-derived biomarkers for diagnosis, staging, treatment, and prognosis [2], [3].

Circulating neoplastic cells disseminated from solid tumors are valuable biomarkers reflecting the disease transcriptome, genome, and proteome. Circulating tumor cells (CTCs) are cells disseminated from the tumor, shed into peripheral blood, and capable of recapitulating tumorigenesis. In epithelialderived tumors, CTCs are phenotypically defined as large cells (30 $\mu \mathrm{m}$ on average) lacking expression of the pan-leukocyte marker, CD45, and expressing cytokeratin (CK), epithelial cell adhesion molecule (EpCAM), or E-cadherin (ECAD) [4][5]. CTC enrichment and isolation technologies exist, including the FDAapproved CellSearch ${ }^{\circledR}$ technology, to enumerate and phenotype CTCs to guide clinical prognosis [6]. With estimates of one CTC per $10^{6}-10^{8}$ leukocytes found in peripheral blood of pancreatic ductal adenocarcinoma (PDAC) patients, levels of these rare biomarkers challenge their functional utility, thus improved approaches to capturing these rare cells are needed [7],[8]. Many groups have focused on using microfluidic sorting, density-based devices, size exclusion filtration, or marker specific isolation methods[9]-[11]. These technologies rely on either a single physical property (size, density) or on specific protein expression (EpCAM, ECAD, CK) to differentiate CTCs from peripheral blood mononuclear cells (PBMCs). However, the rarity of CTCs has greatly limited their application in providing multi-omics information that encompasses tumor heterogeneity and has not translated well into widespread clinical use. 
In the past 5 years, other promising types of unconventional circulating neoplastic cells have been described such as tumor hybrid cells (THC) and circulating hybrid cells (CHCs)[12], [13]. CHCs are described as having hybrid phenotypes of a neoplastic cell and a leukocyte with dual expression of CD45 and tumor markers such as EpCAM or CK[10], [13], [14]. In PDAC patients, CHCs have shown better prognostic value than CTCs as their enumeration correlated with stage and survival [15]. While CHCs are more abundant, their physical and phenotypic similarity to PBMCs makes their isolation and analysis a challenge [8], [16]-[20].

To address these challenges, we developed a microfluidic device utilizing the intrinsic dielectrophoretic (DEP) properties of cells to enable their label-free enrichment, in a fashion compatible with both phenotypic and genotypic downstream analyses. When cells are exposed to this electric field, differential polarization is created between the cells and the surrounding media translating into a resultant force[21], [22]. This resultant force can either be positive DEP where cells travel up the electric field (E-field) gradient, or negative DEP where cells travel down the gradient[23]. The overall magnitude of the DEP force is dictated by the E-field gradient and the particle/cell geometry. Instead, the directionality of the force is determined by relative permittivity between the cell and the surrounding media and is specifically explained by the Clausius-Mossotti factor [24].

DEP as a cell differentiation approach can be grouped into two main categories, batch mode or continuous mode processing. Batch mode processing relies on a unique frequency cut-off where a cell's DEP response changes between positive and negative, for each discrete cell type. Defined frequencies can then be used to separate two cell groups to positive or negative DEP regimes followed by flushing out the negative DEP partition. This approach provides enhanced specificity for discrete cell types expressing narrow dielectrophoretic variance, however it's utility is impeded by low throughput and narrow search space [25]-[30]. In contrast, continuous mode processing uses a secondary force (flow, acoustic or others) to incrementally bias cell trajectory over a period of time[31]-[33]. The combination of continuous flow and DEP can result in multiple cell sorting outcomes in high cell density environments translating into a versatile high-throughput methodology for mixed cell populations with variant dielectrophoretic responses. The majority of strategies currently employed for rare cell enrichment are reliant on clearly defined physical properties and minimal phenotypic variation of the target cell population. However, this approach fails for heterogeneous cell populations with high phenotypic and DEP variance making it unlikely for a single condition to be successful. To address these limitations, we designed and optimized a continuous mode DEP based microfluidic device to allow for enrichment of highly heterogenous rare cell populations like CHCs. The workflow was validated using charged beads, optimized on cell lines and PBMCs, then tested on specimen from cancer patients. In this work, PBMCs from a small amount of blood $(2 \mathrm{~mL})$ are sorted in a high-density environment where non-target immune cells are depleted from the sample enriching for neoplastic cells in a label-free manner. As a proof of concept, enriched $\mathrm{CHCs}$ were subjected to phenotypic and genotypic downstream analysis demonstrating clinically relevant KRAS mutation status in PDAC tumors. 


\section{Results And Discussion}

\section{Design \& Validation Strategy of microfluidic DEP sorting device}

Design. The concept of enriching a target cell population by depleting non-relevant cells is shown schematically in Fig. 1. First, the sample is infused into the microfluidic DEP sorting device, composed of a microfluidic channel and interdigitated electrode arrays. The microfluidic device was manufactured by standard photolithographic and lift-off techniques where a silicon substrate was patterned with an interdigitated electrode layer and a polydimethylsiloxane (PDMS) layer was bonded on top to form a fluidic channel. To allow cells throughout the channel's height to be acted upon by DEP forces, the chamber height was designed to be $25 \mu \mathrm{m}$ or approximately twice the average size of PBMCs. Under continuous flow the sample is centrally confined by two sheath flows providing small variation of initial speed and position. The interdigitated electrodes were designed to have a width and spacing of $35 \mu \mathrm{m}$, approximately 2-3 times the size of PBMCs, to minimize cells being captured on the electrodes by DEP forces (Fig. S1). The V-shape electrode geometry was designed to deflect PBMCs in a high cell density environment by utilizing DEP forces to deflect cells along the electrodes while flow forces direct cells along the length of the device[34]-[36]. Cells experiencing strong DEP forces are depleted to the side outlets, while less responsive cells are enriched in the center channel (Fig. 1A). In order to maximize the DEP response and minimize electrode corrosion, a low conductive buffer at $145 \mathrm{mS} / \mathrm{m}$ (approximately $0.1 x$ PBS) was used during cell enrichment processing for both the sample and sheath inlets. The buffer was chosen to balance cell viability, DEP reactivity, and electrode corrosion[32], [37]. For ease of use and rapid troubleshooting the complete setup was placed under a microscope for continuous viewing where the fluidic channel is visible under a standard $5 x$ objective lens. This setup allows for sample compatibility with genotypic or phenotypic downstream analysis (Fig. 1A) which can be helpful for understanding cellular clinical significance[38].

Validation. To demonstrate the concept of enriching by depleting a target population we used a sample mix composed of $2 \mu \mathrm{m}$ (blue) and $10 \mu \mathrm{m}$ (green) fluorescent polystyrene beads of identical chemistry (Fig. 1B and 1C). The sample mix was introduced to the inlet of the microfluidic DEP sorting device with $A C$ voltage applied to the interdigitated electrodes, and particle movement was monitored by live imaging. We defined optimal condition as the parameters where one type of particle is enriched by the effect of depleting the other. To find this condition, the frequency was swept from $0.1 \mathrm{MHz}$ to $10 \mathrm{MHz}$ at $0.1 \mathrm{MHz}$ increments at constant voltage. Particles were collected at the outlets and analyzed by flow cytometry.

Under the optimal frequency, both bead populations were closely aligned with the center of the channel while near the sample inlet (Fig 1B, part I), however only the $10 \mu \mathrm{m}$ beads were displaced further downstream by DEP forces revealing particle trajectory bias over time and space (Fig. 1B, part II). Under this condition, $95 \%$ of the $10 \mu \mathrm{m}$ beads were depleted from the center due to the application of strong positive DEP forces, while less than $1 \%$ of the $2 \mu \mathrm{m}$ beads were depleted to the sides (Fig. 1C). Fig. 1C also shows an example at one of the sub-optimal frequencies that only $19.5 \%$ of $10 \mu \mathrm{m}$ were depleted, suggesting that the magnitude of the DEP force was smaller than the dominant flow forces. To confirm 
that DEP forces were the primary driver of bead depletion, the device was run without applied DEP and resulted in $99 \%$ of both bead populations remaining in the center unaffected by drift. This workflow demonstrated that depletion of one population by DEP can result in collection of targets with high purity under proper working parameters. To understand how this applied to a complex biological sample mix, we next tested this process on PBMCs and cell lines.

\section{Optimization of PBMC depletion and hybrid cell line enrichment}

PBMC DEP response characterization. As DEP conditions can be optimized to separate discrete physiologic objects, we set out to establish the optimal condition where hybrid cells could be enriched from PBMCs. Cells are biologic entities, thus their conductivity, permittivity, and physiological state are the main drivers of differential DEP response [39]. Healthy PBMCs are composed of heterogeneous populations, but tend to be relatively similar in nature across individuals, therefore healthy PBMCs act as an ideal object to be optimized for depletion. PBMCs are mainly comprised of lymphocytes, monocytes, and granulocytes. Of these, T-cells represent the majority (70-80\%) of the total cell number, therefore we hypothesized that depletion of T-cells would be the primary contributor of neoplastic cell enrichment. First, to understand the DEP response of PBMCs as a collective of cells and as individual subpopulations, we conducted the workflow using PBMCs isolated from whole blood $(n=5)$ from healthy subjects. PBMCs were diluted in DEP buffer ( $1 \times 10^{7}$ cells $\left./ \mathrm{mL}\right)$, and $1 \times 10^{6}$ cells were loaded into the central inlet for each parameter, then subjected to device processing for at least 30 minutes. Cells were not responsive to DEP at low frequencies, thus we focused analyses when cells were visually responsive, between 13 and 17 $\mathrm{MHz}$ at a constant voltage of $8 \mathrm{Vpp}$. We selected this voltage because cells were minimally reactive below $7 \mathrm{Vpp}$ but were trapped on the electrodes at voltages greater than $9 \mathrm{Vpp}$. For each frequency, cells were collected from all three outlets, then stained with antibodies against CD45 (pan-leukocyte), CD3 (Tcell receptor), CD14 (macrophage), and CD19 (pan B-cell) (Fig. 2A). Depletion of each cell type was calculated by flow cytometry analysis of the outlets where the percent of $\mathrm{CD} 3^{+}, \mathrm{CD} 14^{+}$, or $\mathrm{CD} 19^{+}$cells in the side channels was divided by the percent of the cell of interest from the total cells processed (CD $\left.45^{+}\right)$. A similar trend was observed for all populations, where $15 \mathrm{MHz}$ resulted in maximum depletion from the center channel, which was most pronounced for $\mathrm{CD}^{+}$cells $(99.3 \%)$, followed by CD $14^{+}$cells $(92.5 \%)$, and CD19 ${ }^{+}$cells $(88.5 \%)$. Ultimately, only $1.5 \%$ of the initial number of PBMCs $\left(C D 45^{+}\right)$were collected in the central outlet (Fig. 2A). Of the subpopulations, we found $\mathrm{CD}^{+} \mathrm{T}$-cells were the most responsive and had similar depletion levels between 14,15 , and $16 \mathrm{MHz}$ at $97 \%, 99.3 \%$, and $98.3 \%$, respectively. In contrast, depletion of $\mathrm{CD}_{19}{ }^{+}$and $\mathrm{CD} 14^{+}$cells significantly changed from $13 \mathrm{MHz}$ to $15 \mathrm{MHz}$, suggesting that these cell populations were more sensitive to specific DEP parameters. We corroborated the previous findings by Gascoyne et al. that different cell types have different DEP response profiles [40]-[42]. Not only did we observe PBMCs depletion, we found that the PBMCs composition changed post-depletion e.g. there was 7.3-fold and 4.6-fold enrichment of $\mathrm{CD} 19^{+}$and $\mathrm{CD} 14^{+}$cells in the center channel, respectively. These results suggest that across PBMC phenotypes there is a cell type-dependent DEP response that supports their differential separation and that depletion of $\mathrm{CD}^{+}$cells could drive enrichment of rare cells. 
In-vitro CHC DEP response characterization. Next, to understand the DEP response of malignant cells we selected an established in-vitro hybrid cell line generated through the fusion of B16F10 and bone marrowderived macrophages (B16Mf-RFP) as a model of CHCs[13], [43]. In addition, a breast cancer cell line, MCF7, were used as a surrogate of traditional CTCs. Previously, Gast et al. characterized the phenotypic hybrid nature of in vitro derived CHCs. Conveniently these hybrids co-expressed nuclear RFP and cytoplasmic YFP facilitating their differentiation from the MCF7-GFP cells by flow cytometry [12]. To optimize conditions for hybrid cell enrichment, cancer cells were diluted in DEP buffer at $1 \times 10^{7} / \mathrm{mL}$ and only $1 \times 10^{6}$ cells were loaded into the microfluidic DEP sorting device for each parameter. To visualize cancer cell DEP response, fluorescent images were taken at three locations (Fig. 2B), near the inlet (part I), the middle after the first batch of electrodes (part II), and close to the outlets (part III). At $15 \mathrm{MHz} / 8 \mathrm{Vpp}$, both B16Mf-RFP and MCF7-GFP were aligned with the central inlet flow. As the cells flowed through the device, the majority of MCF7 cells depleted to the sides while the majority of B16Mf remained in the center. To quantify each sorting parameter, we processed cells in the device for at least 30 minutes and analyzed all the outlets by flow cytometry. Across all tested frequencies MCF7 cells were more responsive than B16Mf cells. MCF7 cells had a minimum response at $13 \mathrm{MHz}$ where $65 \%$ of the cells were depleted and a maximum response at $16 \mathrm{MHz}$ where $92 \%$ were depleted to the sides (Fig. 2C). In contrast, the minimum DEP response for B16Mf was at $19 \mathrm{MHz}$ where only $6 \%$ of the cells were depleted and the maximum DEP response was at $15 \mathrm{MHz}$ where only $29.5 \%$ of cells were depleted suggesting that hybrid cells have a unique non-responsive DEP profile (Fig. 2C). In parallel, we analyzed the DEP response of healthy PBMCs $\left(C D 45^{+}\right)$under the same conditions. Again, PBMCs were highly responsive across all frequencies with a minimum response at $19 \mathrm{MHz}$ with $77 \%$ of the cells depleted and a maximum response at $15 \mathrm{MHz}$ with $96.5 \%$ of the cells depleted from the center channel (Fig. $2 \mathrm{C}$ ).

Overall, we found a larger differential response between B16Mf-RFP and PBMCs as compared to MCF7GFP cells and PBMCs despite their close proximity in size to PBMCs suggesting that label free sizeindependent enrichment was possible within our workflow. This also suggests that B16Mf-RFP cells inherently have different dielectric properties likely resulting from their hybrid nature. Although numerous studies focus on CTC enrichment and isolation, these strategies rely on either the inherent large size of CTCs or specific membrane protein expression. CHCs are a promising alternative as they are more abundant than CTCs, but there is a lack of technologies focused on their enrichment, a critical factor in developing $\mathrm{CHCs}$ as a cancer biomarker. This data highlights the potential of using DEP to enrich for $\mathrm{CHCs}$ and potentially other rare cellular biomarkers that are difficult to differentiate phenotypically.

Hybrid cell line spiked into healthy_peripheral blood. A number of technologies to study rare cells relies on isolation of single cells, which is challenging. Recently, development of high-throughput single cell technologies allows for analysis of thousands of cells and demonstrates the need for rare cell enrichment rather than pure isolation. For most enrichment strategies there is a tradeoff between total enrichment and overall cellular biomarker loss during processing due to a limit of detection (LOD) threshold. To find the optimal frequency and voltage parameters to enrich for $\mathrm{CHCs}$, we evaluated the enrichment of B16MfRFP cells from PBMCs. PBMCs were isolated from healthy subjects were spiked with 50,000 B16Mf cells 
(50,000 per $1 \times 10^{6}$ PBMCs), then subjected to DEP for at least 30 minutes, and analyzed for cells types isolated from all outlets by flow cytometry. Enrichment of hybrids in the center channel and depletion in the side channels was calculated using flow cytometric data for each outlet (see equations shown in Fig. 2D). This process was repeated for six frequencies, from 13-18 MHz and three voltages for each frequency, 7-9 Vpp. A heatmap was generated to assess center channel hybrid enrichment (fold change) and side channel hybrid loss (percentage loss) taking into account both the frequency and voltage applied to the device (Fig. 2E \& 2F). The enrichment varied from 1.4-fold and 2.8-fold at $13 \mathrm{MHz} / 7 \mathrm{Vpp}$ and $19 \mathrm{MHz} / 9 \mathrm{Vpp}$, respectively, to 18.6 -fold and 12.7 -fold at $15 \mathrm{MHz} / 8 \mathrm{Vpp}$ and $15 \mathrm{MHz} / 9 \mathrm{Vpp}$, respectively. The highest B16Mf enrichment identified was 18.6-fold $(n=5)$ at $15 \mathrm{MHz} / 8 \mathrm{Vpp}$ (Fig. 2E). The voltage was a strong driver of the magnitude of DEP response with greater loss of B16Mf cells to the sides at higher voltages (Fig. 2F). For example, at $15 \mathrm{MHz}$, there was $6.5 \%$ loss to the sides at $7 \mathrm{Vpp}$, $18.6 \%$ at $8 \mathrm{Vpp}$, and $22.7 \%$ at $9 \mathrm{Vpp}$. Overall, we found that $15 \mathrm{MHz}$ and $8 \mathrm{Vpp}$ provided optimal DEP parameters for B16Mf hybrid enrichment and potentially for patient-derived $\mathrm{CHCs}$. These trends observed follow previously describe bell-shaped DEP response curves [44]. In these models as well as in the data presented here, a narrow range of maximum DEP response can be appreciated. Overall, our unique strategy to deplete healthy PBMCs from the sample underlies enrichment of the target tumor cell population.

According to current literature there are approximately $30 \mathrm{CHCs}$ per 500,000 PBMCs in PDAC patients representing only $0.006 \%$ of total cells in blood[14]. This overall rarity highlights a clear need for $\mathrm{CHC}$ enrichment for downstream analyses such as mutational profiling using ddPCR or genomic surveying using single cell sequencing, both of which have a limit of detection (LOD) of $0.1 \%$ [45]-[48]. With 18.6fold enrichment using the described workflow, the microfluidic DEP sorting device described could enrich above $0.1 \%$ LOD suggesting there is utility targeting the depletion of healthy PBMCs to facilitate a more comprehensive understanding of poorly understood circulating pathogenic cells.

Finally, since a strong electric field can induce cell death, which would disrupt downstream analyses, we assessed cell viability throughout the DEP workflow. Non-viable cells can lead to aggregation and clogging, which reduces device performance and sensitivity for isolating cellular biomarkers. The low conductive DEP buffer was designed to maintain cell viability while allowing for DEP separation. In order to study cell viability, the effect of DEP buffer alone and under applied DEP was tested. A549 cells were placed in DEP buffer and cell viability was assessed over time using propidium iodide staining via flow cytometry. A549 cells had $98 \%$ viability after 30 minutes in DEP-buffer and $97 \%$ after 120 minutes (Fig. S2). To measure cell viability post DEP processing at $15 \mathrm{MHz} / 8 \mathrm{Vpp}$, PBMCs were stained with calcien AM from the outlets and immediately measured via flow cytometry (Fig. S2). The side outlets had 99\% viability while the center outlet had $97 \%$ viability, suggesting that enrichment via DEP is compatible with analytic techniques that require cell viability, morphology, and nucleic acid integrity to be maintained.

\section{Label-free Enrichment of KRAS mutant cells from PDAC patients}


To evaluate the ability of the microfluidic DEP sorting device to provide relevant clinical information when assessing peripheral blood from cancer patients, we set out to demonstrate that DEP-enriched CHCs could be used to evaluate KRAS mutational status. Whole blood from four patients with PDAC (stage IIIIV) was collected. All patients had clinically confirmed $K R A S^{\text {mut }}$ primary tumors (Table 1). The majority of PDAC tumors harbor KRAS mutations and its presence in circulation, from circulating cells or cell free DNA, is clinically relevant for cancer detection[49]. Moreover, our group has previously found that a subpopulation of FACS isolated CHCs have KRAS mutation as detected by ddPCR[14]. To confirm the presence of $\mathrm{CHCs}$ in peripheral blood specimen prior to subjection to DEP, PBMCs were isolated from whole blood and analyzed as we have previously reported [13], [14]. Briefly, PBMCs were adhered to glass slides then stained with antibodies against CD45 and CK, and visualized by fluorescence microscopy (Fig. 3A). The size of CHCs $(n=25)$ from all patients was $9.05 \pm 0.89 \mu \mathrm{m}$, and was comparable to the size of PBMCs $(n=25) 8.57 \pm 0.89 \mu \mathrm{m}$, confirming the non-significant difference in size between these two populations (Fig. 3B). As indicated, this negates the possibility of size-based isolation as a valid isolation method for CHCs further supporting the utility of label-free DEP enrichment[13]. Peripheral blood specimen had non-detectable levels of CTCs $\left(\mathrm{CD}_{4} \% / \mathrm{CK}^{+}\right)$by IHC, further supporting the promising nature of $\mathrm{CHCs}$ as an important cancer biomarker due to their detectability and potential for harboring clinically relevant information.

\begin{tabular}{|c|c|c|c|c|c|c|c|c|}
\hline \multicolumn{3}{|c|}{$\begin{array}{l}\text { Patient GenderAge } \\
\text { ID }\end{array}$} & \multirow{2}{*}{$\begin{array}{l}\begin{array}{l}\text { Primary } \\
\text { location }\end{array} \\
\text { Pancreas }\end{array}$} & \multirow{2}{*}{$\begin{array}{c}\begin{array}{c}\text { Intact } \\
\text { primary }\end{array} \\
\text { yes }\end{array}$} & \multirow{2}{*}{$\begin{array}{c}\begin{array}{c}\text { Stage at } \\
\text { collection }\end{array} \\
\text { IV }\end{array}$} & \multicolumn{2}{|c|}{ MetastasisTreatment } & \multirow{2}{*}{$\begin{array}{c}\mathbf{1}^{\circ} \text { Tumor } \\
\text { KRAS }^{\text {mut }} \\
\text { p.G12R }\end{array}$} \\
\hline PDAC3 & $\mathrm{F}$ & 75 & & & & lung & yes & \\
\hline PDAC4 & $\mathrm{F}$ & 75 & Pancreas & yes & III & no & no & p.G12R \\
\hline PDAC1 & $\mathrm{F}$ & 53 & Pancreas & yes & IV & liver & yes & p.G12D \\
\hline PDAC2 & M & 76 & Pancreas & yes & III & no & no & p.G12D \\
\hline
\end{tabular}

Table 1. Patient demographics.

To demonstrate DEP-enriched CHCs harbored mutant KRAS, a smaller aliquot of patient PBMCs were subjected to DEP. A pre-sort aliquot was retained as a control ("Pre-sort", Fig. 3D). Specimens were loaded onto the device $\left(1 \times 10^{6}\right.$ cells) and all samples were processed for at least one hour at $15 \mathrm{MHz}$ and $8 \mathrm{Vpp}$, the optimal conditions for PBMC depletion. Cells were collected from side and center outlets and each channel was subjected to DNA extraction for downstream evaluation of KRAS mutations and total DNA was quantified using Qubit ${ }^{\mathrm{TM}}$ kits (Fig. 3C). Based on DNA concentration we found that an average of $92.6 \% \pm 2.07(n=4)$ of the cells were depleted from the center which is comparable to previous results (Fig. S3) but significantly different than healthy controls ( $p=0.03$, unpaired t test). PBMCs from diseased patients can be influenced by disease resulting in modified proportion of its subpopulation or like immunosenescence where senescent T-cells exhibit abnormal phenotypes [50], [51]. These complex factors may influence PBMC DEP response and lead to the utility of such biomarkers. In this way, optimizing the system to deplete healthy PBMCs in a label-free manner may reveal other disease related cells. 
To demonstrate DEP-isolated CHCs harbor detectible KRAS mutations, we subjected isolated DNA to ddPCR. We used PBMCs of five screened negative subjects as controls. For all samples, ddPCR probes for the seven most common KRAS mutations in PDAC were evaluated. In three out of four patients analyzed, mutant KRAS alleles were identified in cells isolated from the center outlet while the side outlets only contained cells expressing wild-type KRAS (Fig. 3D). For all other outlets and the screen-negative controls, the number of KRAS mutant copies detected were below the LOD for ddPCR [45]-[48]. PDAC3 had the highest number of KRAS mutant copies corresponding to $0.15 \%$ of the total DNA, followed by PDAC4 and PDAC1 with $0.13 \%$ and $0.1 \%$, respectively. PDAC2 had non-detectable levels of KRAS mutations. Although our cohort is limited in number, we identified $K R A S$ mutations in $75 \%$ of evaluated samples ( $p=0.048$, Fisher Exact test), which is comparable to prior studies that only detected $K R A S^{\text {mut }}$ CTCs in $72 \%$ of samples[5].

The average KRAS mutant copies from the three KRAS positive samples was $0.13 \%$. Typically $K R A S$ positive cells are heterozygous therefore $0.26 \%$ of the cells were positive, corresponding to one KRAS mutant cell for every 385 wild-type cells post device processing [52]-[54]. Previously Dietz at al. found an average of $30 \mathrm{CHCs}(n=5)$ for every 500,000 PBMCs in PDAC patients and found that only $9.1 \%$ of $\mathrm{CHCs}$ were KRAS mutant, therefore the enrichment presented here could be as high as 476 -fold. Considering that no CTCs were found by IHC, we believe that KRAS mut cells are likely $\mathrm{CHCs}$ or other important circulating tumor derived cells. The level of enrichment presented here is sufficiently above the LOD for current single cell technologies which could allow for a deeper understanding of circulating cancer cells and associated biology.

\section{Conclusion}

In this study, we present a unique approach addressing the challenge of targeting heterogeneous circulating biomarkers by focusing on healthy PBMCs with defined DEP response to reveal disease specific characteristics. A DEP-driven microfluidic device fabricated with a V-shape electrode design is ideal for such a strategy. Thus, allowing for continuous mode processing of high cell density samples in a short amount of time. The utility of the workflow was optimized for depletion of PBMCs permitted maximal enrichment for a $\mathrm{CHC}$ model cell line. Using this approach, the device was used to process PBMCs from four PDAC patients and was coupled with genotypic downstream analysis using ddPCR. This workflow allowed for the identification of isolated cells with KRAS mutations above the limit of the detection in three out of four patients from only $2 \mathrm{~mL}$ of peripheral blood with one hour of sample processing. We demonstrated DEP-isolated CHCs harbored KRAS mutations as a proof of concept, but the overall strategy is applicable to other cancers or diseases with associated cellular biomarkers to provide clinically relevant readouts.

Future work will focus on coupling this technology with droplet based single cell technologies where both phenotypic and genotypic information can be studied simultaneously[55], [56]. With further biological insights into these potential cellular biomarkers we aim to develop clinical assays beyond KRAS mutation status. With the short sample processing time, low input volume, device reproducibility, and compatibility 
with downstream analytics, our method of cell enrichment by DEP shows promise as a clinical technology with the potential to improve current liquid biopsy strategies of cancer diagnosis and treatment monitoring.

\section{Materials And Methods}

\section{Fabrication and Operation of microfluidic DEP sorting device}

The microfluidic DEP sorting device described here consists of two layers: a silicon chip with an interdigitated patterned electrode layer and a microfluidic PDMS chamber bonded through plasma treatment and heat curing. The bottom layer consists of three parts, a silicon substrate with $500 \mathrm{~nm}$ of wet-growth silicon dioxide $\left(\mathrm{SiO}_{2}\right)$ on both sides, and $90 \mathrm{~nm}$ thick of e-beam deposited platinum electrodes as the conductive components for DEP actuation, and silicon dioxide passivated the edge of the electrodes. The electrodes were interdigitated-separated into two batches to form multiple independent electrodes pairs to actuate the sample in the device. The edge of the electrode is designed to be $15 \mu \mathrm{m}$ from the wall of flow channel, the silicon dioxide passivation avoided cells being trapped at the region where DEP forces is much stronger than the flow stress. The passivation ensures enough shear force to carry sorted cells along the direction of flow.

The device has two DEP actuation region that are connected to two independent pairs of electrode pads. Each electrode pads has two identical circular areas, one was wired bonded by fast drying silver paint (Ted Pella Inc.) and connected to power amplifier (Model 9260, Tabor Electronics Inc) in series with a waveform generator (33622A, Keysight), the other electrode pad was used to monitor voltage and frequency on the device by connected with an oscilloscope (DSOX2024A, Keysight) at the beginning of each experiment and every time parameters were adjusted. The wired device was place on a water-cooled aluminum heat sink to keep device at or below room temperature. The complete setup was placed under a microscope for real time monitoring.

The microfluidic chamber (Fig. S2) consists of one sample inlet of $0.5 \mathrm{~mm}$ width and two sheath flow inlets of $1 \mathrm{~mm}$ for a total ratio of 4:1 sheath to sample ratio. The total chamber width is $2 \mathrm{~mm}$ which allowed us to view the entire channel within a field of view by a $5 x$ fluorescence microscope objective as shown in Fig. 2C. At the outlet, to allow only highly responsive cells to be depleted from the sample, we design the center outlet to be wider than the top and bottom outlet. The center outlet is $0.9 \mathrm{~mm}$, and the sides are $0.8 \mathrm{~mm}$ each for a total ratio of $16: 9$ sheath to sample. The flow is pulled from all three outlets by two flow-driven syringe pumps, the withdrawal setup provides enhanced flow stability compared to active infusion from the inlets. The center-focused flow was adjusted to a flow rate of $2 \mu \mathrm{L} /$ minute for each of the sheath flow and $2.5 \mu \mathrm{L} /$ minute for the center outlet.

\section{Microfluidic device preparation and assembly}

Polydimethylsiloxane (PDMS, Sylgard 184, Dow Corning, MI) was prepared as the standard 10:1 ratio and cured at $65{ }^{\circ} \mathrm{C}$ for at least $2 \mathrm{~h}$ and cooled down at room temperature before use. Then, inlets were 
punctured with $2 \mathrm{~mm}$ biopsy puncture (Ted Pella) and outlets were puncture with $1 \mathrm{~mm}$ biopsy puncture (Ted Pella). The PDMS part was cleaned by sonication in isopropanol, then PDMS chamber was gas dried followed by incubation in the $80^{\circ} \mathrm{C}$ oven for at least 15 minutes prior to assembly. On the other hand, the electrode patterned silica wafer was sequentially cleaned by acetone, methanol, isopropanol and deionized water. The device was dried by pressurized nitrogen gas prior to assembly.

Both the PDMS microfluidic chamber and silicon chip were treated with oxygen plasma (35W, 600mtorr) for 1 minute. The PDMS chamber was aligned to the fiducial on the silicon chip under a stereoscope and placed on a hot plate at $95^{\circ} \mathrm{C}$ for 60 minutes followed by air cool until room temperature. After PDMSsilicon chip bonding process, 21-gauge wire was wired to the electrode pad using fast drying silver paint (Ted Pella Inc.) and left at room temperature for 1 hour before incubated at $80^{\circ} \mathrm{C}$ for 1 hour to fully dry the silver paint. The 5 minutes epoxy was applied on the electrode pad and silver paint to enhance mechanical strength of the wiring. The device was incubated at $80^{\circ} \mathrm{C}$ for more 30 minutes and cooled down to room temperature prior to the experiment.

For each experiment, $20 \mathrm{~cm}$ PTFE tubing (24 AWG, Allied Electronics) was connected to a $1 \mathrm{~mL}$ syringe. In addition, $0.5 \mathrm{~cm}$ tygon tubing (OD: $2.286 \mathrm{~mm}$, ID: $1.270 \mathrm{~mm}$ ) was plugged into the inlets of the device, a 1 $\mathrm{mL}$ pipette was cut approximately in half and inserted on the inlet tubing of the side channels to create a reservoir of sheath flow. A $200 \mu$ pipette was inserted on the tubing on the center to create a sample reservoir. Finally, the device was placed on a recirculating water-cooled aluminum fixture, and monitored by a microscope.

\section{DEP Buffer}

To make DEP buffer, $90000 \mathrm{mg}$ of sucrose (Thermo Fisher Scientific), $12086 \mathrm{mg}$ of dextrose (Thermo Fisher Scientific), and $2000 \mathrm{mg}$ of bovine serum albumin (BSA) was dissolved in deionized water (18.2 MOhm-cm) with the total volume of $900 \mathrm{~mL}$. $95 \mathrm{~mL}$ of Dulbecco's modified Eagle's Media (DMEM) supplemented with $10 \%$ fetal bovine serum (FBS) was then added to make a final volume of $950 \mathrm{~mL}$ solution. The conductivity of the buffer was adjusted to $145 \mathrm{mS} / \mathrm{m}$ by adding DMEM with $10 \%$ FBS supplement. The final solution was filtrated by $0.22 \mu \mathrm{m}$ filter and preserved in $4{ }^{\circ} \mathrm{C}$.

\section{Bead Sorting}

Prior to each experiment DEP buffer was placed under vacuum for at least 30 minutes to degas it. $10 \mu \mathrm{m}$ FluoSpheres ${ }^{\text {TM }}$ Polystyrene Microspheres and $2.0 \mu \mathrm{m}$ FluoSpheres ${ }^{\text {TM }}$ Carboxylate-Modified Microspheres were diluted to a total of $1 \times 10^{6}$ beads. The bead mixture was then centrifuged and suspended on $100 \mu \mathrm{l}$ of degassed cold DEP buffer. This solution was then introduced on the microfluidic device and different conditions as described on Fig. $1 \mathrm{C}$ were applied. For each condition, the solution was processed for at least 30 minutes, outlets collected, measured using BD FACS Symphony and analyzed with FlowJo.

\section{Cell culture}


MCF7 (breast adenocarcinoma) were purchased from American Type Culture Collection (ATCC, Manassas, VA) and maintained in Dulbecco's modified Eagle's Media (DMEM) supplemented with 10\% fetal bovine serum (FBS) and 1\% Penicillin Streptomycin Glutamine (PSG). B16F10-macrophage hybrids (B16Mf) were previously generated and maintained in Dulbecco's modified Eagle's Media (DMEM) supplemented with $10 \%$ fetal bovine serum (FBS) and 1\% Penicillin Streptomycin Glutamine (PSG)[13].

\section{Cell line PBMC DEP analysis}

Prior to each experiment DEP buffer was placed under vacuum for at least 30 minutes to degas it. Deidentified healthy participant whole blood was obtained from the CEDAR repository following IRB protocol into either a Heparin (BD367874) or ACD (BD364606) vacutainer tubes. Within 15 minutes of the blood draw, 8-10 mL of whole blood was processed following the SepMate ${ }^{\mathrm{TM}}$ (STEMCELL Technologies, cat\# 85450) protocol. Cells were counted using the Countess ${ }^{T M}$ II (Applied Biosystems) automated cell counter and diluted to $1 \times 10^{7}$ cells $/ \mathrm{mL}$ in cold PBS and stored on ice. Cell lines were trypsinized, washed, and reconstituted in DEP buffer. Prior to loading into the DEP device, $100 \mu$ l of cell solution was diluted to $1 \mathrm{~mL}$ in DEP buffer, centrifuged for 5 minutes at $300 \mathrm{~g}$, and reconstituted in 60-80 $\mu$ DEP buffer.

Reconstituted cells were loaded into the center channel inlet and collected for at least 30 minutes at each specific frequency and voltage combination. In the case of the spiking experiments, 50,000 B16Mf cells were added for per $1 \times 10^{6}$ PBMCs prior to loading into the device. Each outlet was collected, transferred to standard $5 \mathrm{ml}$ round bottom polystyrene flow cytometry tubes, stained with AF647-CD45(BioLegend, clone H130), PE-CD3 (BioLegend, clone HIT3a), AF488-CD19 (eBioscience, clone HIB19) and V450-CD14 (BD Horizon, clone $\mathrm{M} \varphi \mathrm{P} 9$ ) on ice for at least 30 minutes, measured on $\mathrm{BD}$ flow cytometry Symphony, and analyzed with FlowJo.

\section{Viability testing}

To measure the viability of cells in DEP buffer alone, A549 cells we placed in either DMEM or DEP buffer on ice and serially sampled every 30 minutes. Each sample was stained with propidium iodide $(\mathrm{PI})$, measured on BD flow cytometry Symphony, and analyzed with FlowJo. $\mathrm{Pl}^{+}$cells were considered dead or dying. To measure the viability of cells post-DEP sorting through the device, cells were collected at each outlet and stained with Calcien AM (Invitrogen ${ }^{\text {TM }}$ C3099). As before, cells were flow cytometry analyzed and Calcein AM positive cells were considered viable.

\section{Clinical Sample Processing for immunofluorescence.}

For evaluation of $\mathrm{CHCs}$, samples were first treated with a $5 \%$ bovine serum albumin solution, followed by TrueBlack Lipofuscin Autofluorescence Quencher (Biotium), and Image-iT FX Signal Enhancer (Invitrogen), then stained with fluorescent-conjugated antibodies for pan-cytokeratin (CK; eBioscience, clone: AE1/AE), and CD45 (Biolegend, clone:HI30), and counterstained with the nuclear dye, DAPI. Each sample was processed with unstained and isotype controls (eBioscience). Specimens were digitally imaged with a Zeiss AxioScanner. PBMC and $\mathrm{CHC}$ size was measure using the Zen software ruler. 


\section{Clinical Sample Processing for DEP device}

Deidentified samples were retrieved from Oregon Health \& Science University (OHSU) under the Oregon Pancreas Tissue Registry and the Brenden-Colson Center for Pancreatic Care. Informed consent was obtained from all subjects. All experimental protocols were approved by the OHSU Institutional Review Board. All methods were carried out in accordance with relevant guidelines and regulations. At least 1.5 $\mathrm{mL}$ of whole blood was processed immediately using SepMate ${ }^{\mathrm{TM}}$ (STEMCELL Technologies, cat\# 85450) protocol. Cells were counted using Countess ${ }^{\mathrm{TM}}$ II (Applied Biosystems) automated cell counter and diluted to $1 \times 10^{7}$ cells $/ \mathrm{mL}$ in cold DEP buffer and stored on ice. As previously described the chip was primed with degassed DEP buffer and the voltage/frequency parameters were set prior to sample loading. The total sample $(60-100 \mu \mathrm{L})$ was loaded onto the chip and processed for at least 30 minutes but not more than 60 minutes.

\section{DNA extraction and droplet digital PCR}

After all outlets were collected samples were centrifuges at $800 \mathrm{~g}$ for 5 minutes and supernatant was removed. Then Pure ${ }^{\mathrm{TM}}$ DNA Extraction Kit was used according to the manufacturer's protocol. Briefly, 155 $\mu \mathrm{L}$ of digestion buffer was added to the lyophilized proteinase $\mathrm{K}$ and resuspended by pipetting up and down for at least 20 times. $150 \mu \mathrm{L}$ of that solution was added to the cell pellets of every outlet and incubated at $65^{\circ} \mathrm{C}$ for at least 3 hours. After incubation, Ampure XP beads with a $1.2 x$ ratio $(\mathrm{v} / \mathrm{v})$ was added and DNA was isolated according to the manufacturer's protocol. Briefly, samples were under rotation for 10 minutes prior to magnetic separation of beads and discard of the supernatant. Then $80 \%$ ethanol was added to the tube, with samples still in the magnetic rag, and incubated for 30 seconds before discarding the supernatant. This process was repeated twice and then 11 microliters of water were added to the beads and resuspended. Samples were incubated for 10 minutes prior to collection of DNA, 10 microliters total, that was either used immediately or store at $-20^{\circ} \mathrm{C}$. ddPCR was performed using the ddPCR $^{\text {TM }}$ KRASG12/G13 Screening Kit \#1863506 (Bio-Rad Laboratories, CA). Droplets were generated with the Auto Droplet Generator (Bio-Rad Laboratories, CA) and measured on the QX200 ${ }^{\mathrm{TM}}$ Droplet Reader (Bio-Rad Laboratories, CA). PCR parameters were set according to manufacturer recommendations, $95^{\circ} \mathrm{C}$ for 10 minutes, followed by 40 cycles of $94^{\circ} \mathrm{C}$ for $30 \mathrm{~s}$ and $55^{\circ} \mathrm{C}$ for 1 minute, followed by 10 minutes of $98^{\circ} \mathrm{C}$. Mutant and wild type KRASthresholds were set to $\geq 99 \%$ of mutant (A549 cell line) and wild type (A375 cell line) controls were positive and $100 \%$ of blanks were negative.

\section{Declarations}

\section{Acknowledgments}

This project was supported by funding from the Cancer Early Detection Advanced Research (CEDAR) center at Oregon Health \& Science University's Knight Cancer Institute. The authors would like to thank the CEDAR Repository, the Brenden-Colson Center for Pancreatic Care at OHSU and the Oregon Pancreas Tissue Registry. KTG would like to thank the Achievement Rewards for College Scientists (ARCS) 
Foundation Oregon for their continued financial support. BSW, was partially supported by the Collins Foundation Trust Grant and Medical Research Foundation Grant. The authors declare no conflict of interests.

\section{References}

[1] H. Wang et al., "Global, regional, and national life expectancy, all-cause mortality, and cause-specific mortality for 249 causes of death, 1980-2015: a systematic analysis for the Global Burden of Disease Study 2015," Lancet, vol. 388, no. 10053, pp. 1459-1544, Oct. 2016, doi: 10.1016/S0140-6736(16)310121.

[2] J. Wang, S. Chang, G. Li, and Y. Sun, "Application of liquid biopsy in precision medicine: opportunities and challenges," Frontiers of Medicine, vol. 11, no. 4. Higher Education Press, pp. 522-527, Dec. 01, 2017, doi: 10.1007/s11684-017-0526-7.

[3] S. Perakis and M. R. Speicher, "Emerging concepts in liquid biopsies," BMC Medicine, vol. 15, no. 1. BioMed Central Ltd., pp. 1-12, Apr. 06, 2017, doi: 10.1186/s12916-017-0840-6.

[4] J. Zhou, A. Kulasinghe, A. Bogseth, K. O'Byrne, C. Punyadeera, and I. Papautsky, "Isolation of circulating tumor cells in non-small-cell-lung-cancer patients using a multi-flow microfluidic channel," Microsystems Nanoeng., vol. 5, no. 1, pp. 1-12, Dec. 2019, doi: 10.1038/s41378-019-0045-6.

[5] B. Kulemann et al., "Pancreatic cancer: Circulating Tumor Cells and Primary Tumors show Heterogeneous KRAS Mutations," Sci. Rep., vol. 7, no. 1, pp. 1-11, Dec. 2017, doi: 10.1038/s41598-01704601-z.

[6] L. Wang, P. Balasubramanian, A. P. Chen, S. Kummar, Y. A. Evrard, and R. J. Kinders, "Promise and limits of the CellSearch platform for evaluating pharmacodynamics in circulating tumor cells," Seminars in Oncology, vol. 43, no. 4. W.B. Saunders, pp. 464-475, Aug. 01, 2016, doi: 10.1053/j.seminoncol.2016.06.004.

[7] V. Martini, S. Timme-Bronsert, S. Fichtner-Feigl, J. Hoeppner, and B. Kulemann, "Circulating tumor cells in pancreatic cancer: Current perspectives," Cancers, vol. 11, no. 11. MDPI AG, Nov. 01, 2019, doi: 10.3390/cancers 11111659 .

[8] J. S. Ankeny et al., "Circulating tumour cells as a biomarker for diagnosis and staging in pancreatic cancer," Br. J. Cancer, vol. 114, no. 12, pp. 1367-1375, Jun. 2016, doi: 10.1038/bjc.2016.121.

[9] P. Bankó et al., "Technologies for circulating tumor cell separation from whole blood," Journal of Hematology and Oncology, vol. 12, no. 1. BioMed Central Ltd., pp. 1-20, May 14, 2019, doi: 10.1186/s13045-019-0735-4. 
[10] S. Ribeiro-Samy et al., "Fast and efficient microfluidic cell filter for isolation of circulating tumor cells from unprocessed whole blood of colorectal cancer patients," Sci. Rep., vol. 9, no. 1, pp. 1-12, Dec. 2019, doi: 10.1038/s41598-019-44401-1.

[11] L. Xu et al., "Optimization and Evaluation of a Novel Size Based Circulating Tumor Cell Isolation System," PLoS One, vol. 10, no. 9, p. e0138032, Sep. 2015, doi: 10.1371/journal.pone.0138032.

[12] L. A. Aguirre et al., "Tumor stem cells fuse with monocytes to form highly invasive tumor-hybrid cells," Oncoimmunology, vol. 9, no. 1, Jan. 2020, doi: 10.1080/2162402X.2020.1773204.

[13] C. E. Gast et al., "Cell fusion potentiates tumor heterogeneity and reveals circulating hybrid cells that correlate with stage and survival," Sci. Adv., vol. 4, no. 9, pp. 7828-7840, Sep. 2018, doi: $10.1126 /$ sciadv.aat7828.

[14] M. S. Dietz et al., "Relevance of Circulating Hybrid Cells as a Non-Invasive Biomarker for Myriad Solid Tumors," bioRxiv, p. 2021.03.11.434896, Mar. 2021, doi: 10.1101/2021.03.11.434896.

[15] K. S. Cheng et al., "ALICE: a hybrid Al paradigm with enhanced connectivity and cybersecurity for a serendipitous encounter with circulating hybrid cells," Theranostics, vol. 10, no. 24, pp. 11026-11048, 2020, doi: $10.7150 /$ thno.44053.

[16] Y. Manjunath et al., "Tumor-Cell-Macrophage Fusion Cells as Liquid Biomarkers and Tumor Enhancers in Cancer," Int. J. Mol. Sci., vol. 21, no. 5, p. 1872, Mar. 2020, doi: 10.3390/ijms21051872.

[17] W. T. Yan et al., "Circulating tumor cell status monitors the treatment responses in breast cancer patients: A meta-analysis," Sci. Rep., vol. 7, no. 1, pp. 1-12, Mar. 2017, doi: 10.1038/srep43464.

[18] A. Lucci et al., "Circulating tumour cells in non-metastatic breast cancer: A prospective study," Lancet Oncol., vol. 13, no. 7, pp. 688-695, Jul. 2012, doi: 10.1016/S1470-2045(12)70209-7.

[19] L. Resel Folkersma, L. San José Manso, I. Galante Romo, J. Moreno Sierra, and C. Olivier Gómez, "Prognostic significance of circulating tumor cell count in patients with metastatic hormone-sensitive prostate cancer," Urology, vol. 80, no. 6, pp. 1328-1332, Dec. 2012, doi: 10.1016/j.urology.2012.09.001.

[20] D. L. Adams et al., "Circulating giant macrophages as a potential biomarker of solid tumors," Proc. Natl. Acad. Sci. U. S. A., vol. 111, no. 9, pp. 3514-3519, Mar. 2014, doi: 10.1073/pnas.1320198111.

[21] N. A. Rahman, F. Ibrahim, and B. Yafouz, "Dielectrophoresis for Biomedical Sciences Applications: A Review," doi: 10.3390/s17030449.

[22] T. B. Jones and M. Washizu, "Multipolar dielectrophoretic and electrorotation theory," J. Electrostat., vol. 37, no. 1-2, pp. 121-134, May 1996, doi: 10.1016/0304-3886(96)00006-X. 
[23] K. Khoshmanesh, S. Nahavandi, S. Baratchi, A. Mitchell, and K. Kalantar-zadeh, "Dielectrophoretic platforms for bio-microfluidic systems," Biosensors and Bioelectronics, vol. 26, no. 5. Elsevier, pp. 18001814, Jan. 15, 2011, doi: 10.1016/j.bios.2010.09.022.

[24] P. R. C. Gascoyne, X. B. Wang, Y. Huang, and R. F. Becker, "Dielectrophoretic separation of cancer cells from blood," IEEE Trans. Ind. Appl., vol. 33, no. 3, pp. 670-678, 1997, doi: 10.1109/28.585856.

[25] C. F. Gonzalez and V. T. Remcho, "Harnessing dielectric forces for separations of cells, fine particles and macromolecules," Journal of Chromatography A, vol. 1079, no. 1-2 SPEC. ISS. Elsevier, pp. 59-68, Jun. 24, 2005, doi: 10.1016/j.chroma.2005.03.070.

[26] F. F. Becker, X. B. Wang, Y. Huang, R. Pethig, J. Vykoukal, and P. R. C. Gascoyne, "Separation of human breast cancer cells from blood by differential dielectric affinity," Proc. Natl. Acad. Sci. U. S. A., vol. 92, no. 3, pp. 860-864, Jan. 1995, doi: 10.1073/pnas.92.3.860.

[27] J. Yang, "Cell separation on microfabricated electrodes using dielectrophoretic/gravitational fieldflow fractionation," Anal. Chem., vol. 71, no. 5, pp. 911-918, Mar. 1999, doi: 10.1021/ac981250p.

[28] P. R. C. Gascoyne, J. Noshari, T. J. Anderson, and F. F. Becker, "Isolation of rare cells from cell mixtures by dielectrophoresis," Electrophoresis, vol. 30, no. 8, pp. 1388-1398, 2009, doi:

10.1002/elps.200800373.

[29] H. Esmaeilsabzali, T. V. Beischlag, M. E. Cox, A. M. Parameswaran, and E. J. Park, "Detection and isolation of circulating tumor cells: Principles and methods," Biotechnology Advances, vol. 31, no. 7. Elsevier, pp. 1063-1084, Nov. 15, 2013, doi: 10.1016/j.biotechadv.2013.08.016.

[30] M. Di Trapani, N. Manaresi, and G. Medoro, "DEPArray ${ }^{T M}$ system: An automatic image-based sorter for isolation of pure circulating tumor cells," Cytom. Part A, vol. 93, no. 12, pp. 1260-1266, Dec. 2018, doi: 10.1002/cyto.a.23687.

[31] X. Hu, P. H. Bessette, J. Qian, C. D. Meinhart, P. S. Daugherty, and H. T. Soh, "Marker-specific sorting of rare cells using dielectrophoresis," Proc. Natl. Acad. Sci. U. S. A., vol. 102, no. 44, pp. 15757-15761, Nov. 2005, doi: 10.1073/pnas.0507719102.

[32] A. J. Smith et al., "Rapid cell separation with minimal manipulation for autologous cell therapies," Sci. Rep., vol. 7, no. 1, pp. 1-15, Feb. 2017, doi: 10.1038/srep41872.

[33] P. Balasubramanian et al., "Antibody-independent capture of circulating tumor cells of non-epithelial origin with the ApoStream ${ }^{\circledR}$ system," PLoS One, vol. 12, no. 4, p. e0175414, Apr. 2017, doi: 10.1371/journal.pone.0175414.

[34] X. Nie, Y. Luo, P. Shen, C. Han, D. Yu, and X. Xing, "High-throughput dielectrophoretic cell sorting assisted by cell sliding on scalable electrode tracks made of conducting-PDMS," Sensors Actuators, $B$ 
Chem., vol. 327, p. 128873, Jan. 2021, doi: 10.1016/j.snb.2020.128873.

[35] Y. Wu, Y. Ren, Y. Tao, L. Hou, and H. Jiang, "High-Throughput Separation, Trapping, and Manipulation of Single Cells and Particles by Combined Dielectrophoresis at a Bipolar Electrode Array," Anal. Chem., vol. 90, no. 19, pp. 11461-11469, Oct. 2018, doi: 10.1021/acs.analchem.8b02628.

[36] H. Song et al., "Continuous-flow sorting of stem cells and differentiation products based on dielectrophoresis," Lab Chip, vol. 15, no. 5, pp. 1320-1328, Mar. 2015, doi: 10.1039/c4lc01253d.

[37] A. Y. L. Jiang et al., "High-throughput continuous dielectrophoretic separation of neural stem cells," Biomicrofluidics, vol. 13, no. 6, p. 064111, Nov. 2019, doi: 10.1063/1.5128797.

[38] J. Cheng et al., "Nanotechnology-assisted isolation and analysis of circulating tumor cells on microfluidic devices," Micromachines, vol. 11, no. 8. MDPI AG, p. 774, Aug. 01, 2020, doi: 10.3390/MI11080774.

[39] J. S. Crane and H. A. Pohl, "Theoretical models of cellular dielectrophoresis," J. Theor. Biol., vol. 37, no. 1, pp. 15-41, Oct. 1972, doi: 10.1016/0022-5193(72)90113-0.

[40] S. Shim, K. Stemke-Hale, J. Noshari, F. F. Becker, and P. R. C. Gascoyne, "Dielectrophoresis has broad applicability to marker-free isolation of tumor cells from blood by microfluidic systems," Biomicrofluidics, vol. 7, no. 1, Feb. 2013, doi: 10.1063/1.4774307.

[41] P. R. C. Gascoyne, S. Shim, J. Noshari, F. F. Becker, and K. Stemke-Hale, "Correlations between the dielectric properties and exterior morphology of cells revealed by dielectrophoretic field-flow fractionation," Electrophoresis, vol. 34, no. 7, pp. 1042-1050, Apr. 2013, doi: 10.1002/elps.201200496.

[42] P. R. C. Gascoyne and S. Shim, "Isolation of circulating tumor cells by dielectrophoresis," Cancers (Basel)., vol. 6, no. 1, pp. 545-579, 2014, doi: 10.3390/cancers6010545.

[43] E. Sinkala et al., "Profiling protein expression in circulating tumour cells using microfluidic western blotting," Nat. Commun., vol. 8, no. 1, pp. 1-12, Mar. 2017, doi: 10.1038/ncomms14622.

[44] T. Jiang, Y. Jia, H. Sun, X. Deng, D. Tang, and Y. Ren, "Dielectrophoresis Response of Water-in-Oil-inWater Double Emulsion Droplets with Singular or Dual Cores," Micromachines, vol. 11, no. 12, p. 1121, Dec. 2020, doi: 10.3390/mi11121121.

[45] K. Morita et al., "Clonal evolution of acute myeloid leukemia revealed by high-throughput single-cell genomics," Nat. Commun., vol. 11, no. 1, pp. 1-17, Dec. 2020, doi: 10.1038/s41467-020-19119-8.

[46] L. Dong, S. Wang, B. Fu, and J. Wang, "Evaluation of droplet digital PCR and next generation sequencing for characterizing DNA reference material for KRAS mutation detection," Sci. Rep., vol. 8, no. 1, p. 9650 , Dec. 2018, doi: 10.1038/s41598-018-27368-3. 
[47] “Performance of the Tapestri ® Platform for Single-Cell Targeted DNA Sequencing."

[48] D. Azuara et al., "Nanofluidic Digital PCR for KRAS Mutation Detection and Quantification in Gastrointestinal Cancer," Clin. Chem., vol. 58, no. 9, pp. 1332-1341, Sep. 2012, doi: 10.1373/clinchem.2012.186577.

[49] A. M. Waters and C. J. Der, "KRAS: The critical driver and therapeutic target for pancreatic cancer," Cold Spring Harb. Perspect. Med., vol. 8, no. 9, p. a031435, Sep. 2018, doi: 10.1101/cshperspect.a031435.

[50] W. Ming et al., "Two Distinct Subtypes Revealed in Blood Transcriptome of Breast Cancer Patients With an Unsupervised Analysis," Front. Oncol., vol. 9, p. 985, Oct. 2019, doi: 10.3389/fonc.2019.00985.

[51] J. Lian, Y. Yue, W. Yu, and Y. Zhang, "Immunosenescence: a key player in cancer development," Journal of Hematology and Oncology, vol. 13, no. 1. BioMed Central Ltd, pp. 1-18, Dec. 01, 2020, doi: 10.1186/s13045-020-00986-z.

[52] C. Almoguera, D. Shibata, K. Forrester, J. Martin, N. Arnheim, and M. Perucho, "Most human carcinomas of the exocrine pancreas contain mutant c-K-ras genes," Cell, vol. 53, no. 4, pp. 549-554, May 1988, doi: 10.1016/0092-8674(88)90571-5.

[53] J. Soh et al., "Oncogene mutations, copy number gains and mutant allele specific imbalance (MASI) frequently occur together in tumor cells," PLoS One, vol. 4, no. 10, Oct. 2009, doi: 10.1371/journal.pone.0007464.

[54] A. J. Aguirre et al., "Activated Kras and Ink4a/Arf deficiency cooperate to produce metastatic pancreatic ductal adenocarcinoma," Genes Dev., vol. 17, no. 24, pp. 3112-3126, Dec. 2003, doi: $10.1101 /$ gad. 1158703 .

[55] Tapestri, "Tapestri Single-cell Custom DNA and Protein Panels Uncover genotypic and phenotypic insights simultaneously from single cells," 2020. Accessed: Mar. 16, 2021. [Online]. Available: www.missionbio.com.

[56] J. Qian et al., "A pan-cancer blueprint of the heterogeneous tumor microenvironment revealed by single-cell profiling," Cell Res., vol. 30, no. 9, pp. 745-762, Sep. 2020, doi: 10.1038/s41422-020-0355-0.

\section{Figures}




\section{A}

(1) Collect blood and isolate PBMCs
Enrich for CHCs using a microfluidic dielectrophoretic device OUT

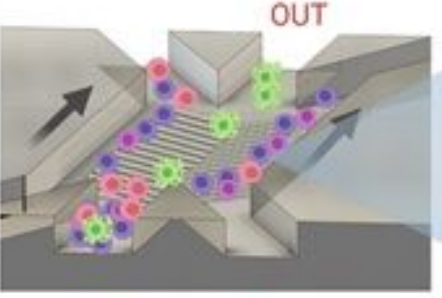

IN 2 chCs $]$ PBMCs

B

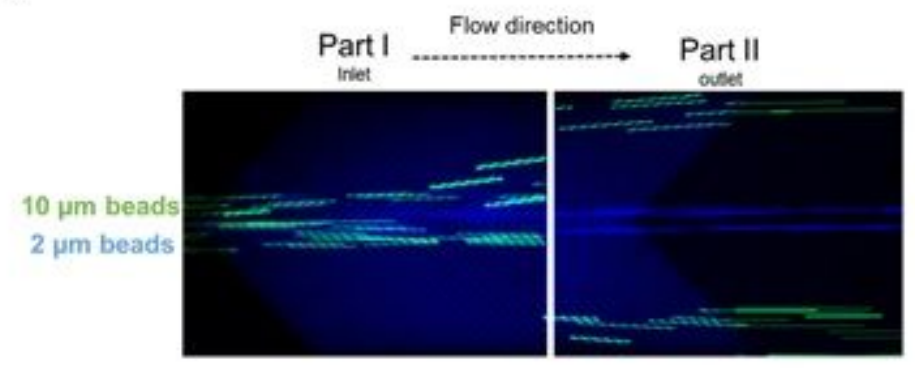

(3) Downstream analysis: Genotype and phenotype
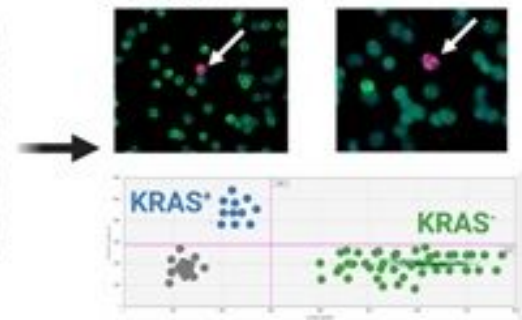

C

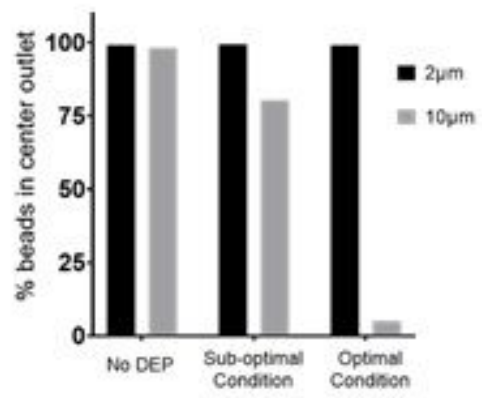

\section{Figure 1}

Workflow for label-free enrichment of unconventional circulating neoplastic cells and proof of concept demonstrating target bead enrichment $(\mathrm{A})$ Schematic representation of $\mathrm{CHC}$ enrichment from patient blood. (1) PBMCs are isolated from $2 \mathrm{ml}$ of whole blood. (2) DEP microfluidic device is used to enrich for $\mathrm{CHCs}$ and samples is collected from outlets. (3) Downstream analysis, both phenotypic and genotypic, can be performed to obtain clinically relevant information. Created with BioRender.com. (B) Fluorescent images of microfluidic channel showing differential response of polystyrene beads, $10 \mu \mathrm{m}$ (green) and 2 $\mu \mathrm{m}$ (blue). Part I represents the inlet and part II represent the outlet. (C) Percent of beads collected from the center outlet at an optimal condition ( $5 \mathrm{MHz})$, a sub-optimal condition $(300 \mathrm{kHz})$, and with no applied DEP.

A

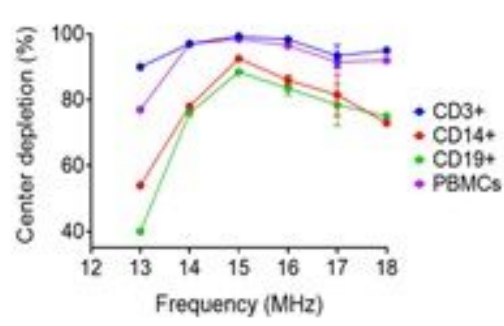

D

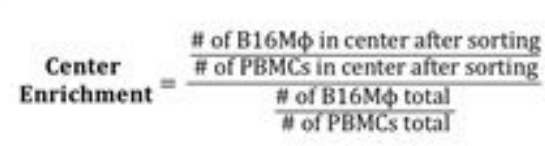

Side Loss $=\frac{\# \text { of } B 16 \mathrm{M} \phi \text { in sides after sorting }}{\# \text { of } \mathrm{B} 16 \mathrm{M} \phi \text { total }}$
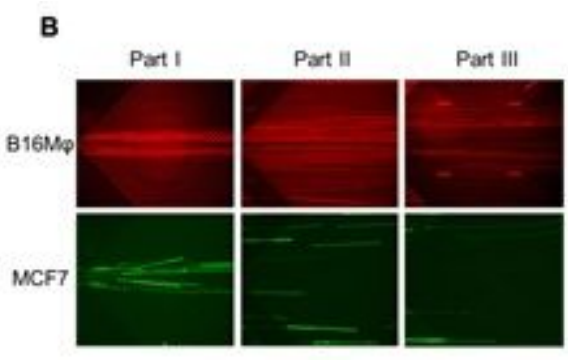

$\mathbf{E}$

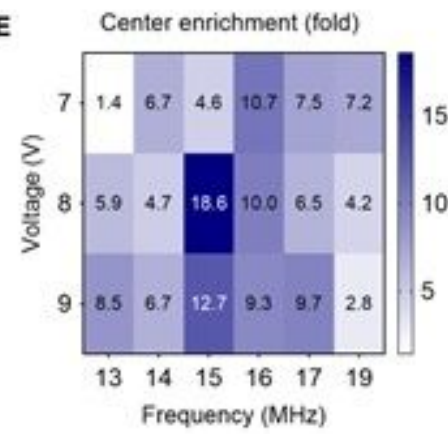

C

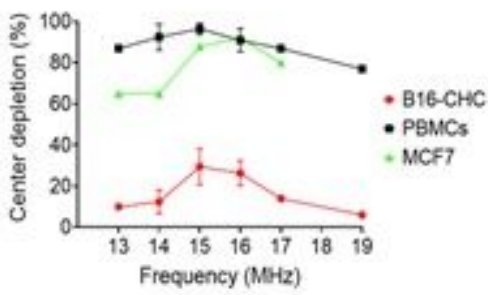

$\mathbf{F}$

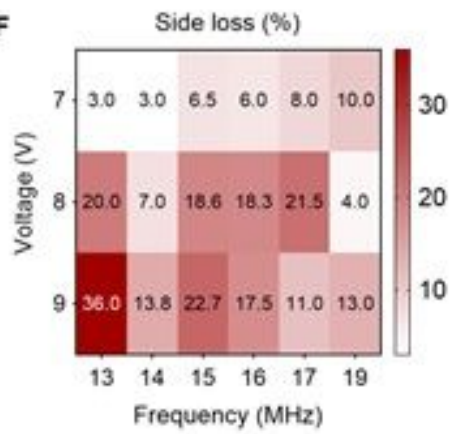


Figure 2

DEP response characterization of PBMCs from healthy donors and cancer cell lines. (A) PBMC subpopulation analysis as presented by depletion of CD3+, CD14+, CD19+, and CD45+ cells across frequencies, $13 \mathrm{MHz}$ to $18 \mathrm{MHz}$ at a constant $8 \mathrm{Vpp}$ by flow cytometry. (B) Fluorescent images near the inlet (Part I), at the center between batches of electrodes (Part II), and close to the outlet (Part III) of the microfluidic channel revealed depletion of MCF7 (green) and less reactive B16Mf (red) at $15 \mathrm{MHz}$ and 8 Vpp. (C) Percent depletion of B16Mf, MCF7, and PBMCs from the center outlet across frequencies, 13 $\mathrm{MHz}$ to $19 \mathrm{MHz}$, at a constant $8 \mathrm{Vpp}$ by flow cytometry. (D) Equations for center enrichment and side loss used for $(E)$ and $(F)$. (E) Center enrichment heatmap with voltage $(V)$ in the $y$ axis and frequency $(\mathrm{MHz})$ in the $x$ axes $(n=1-5)$. (F) Percentage side loss heatmap with voltage $(V)$ in the $y$ axis and frequency $(M H z)$ in the $x$ axes $(n=1-5)$.

a)

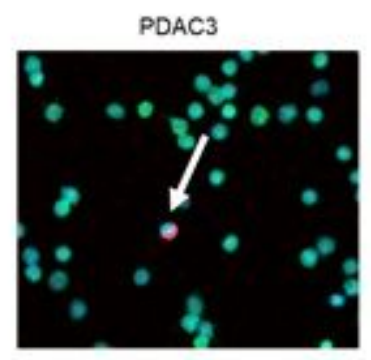

b)

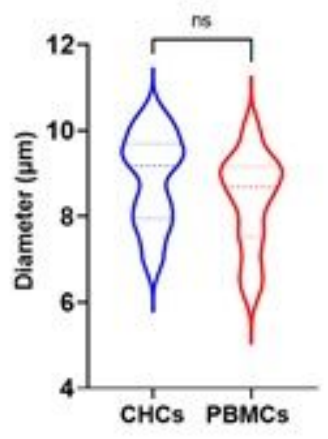

c)

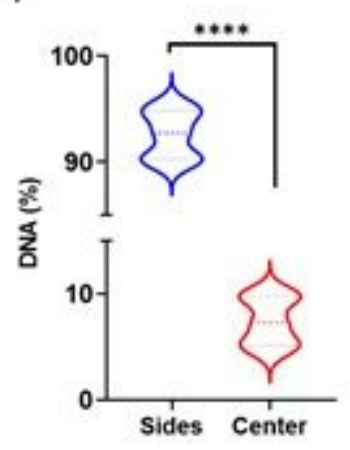

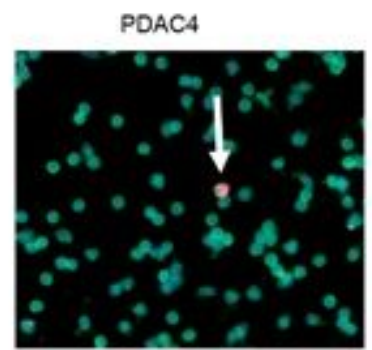

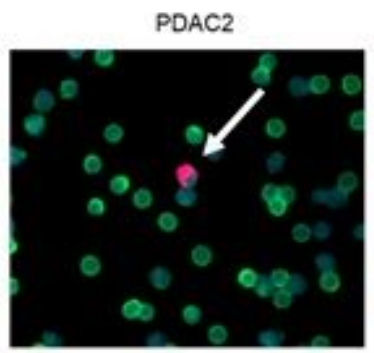

d)

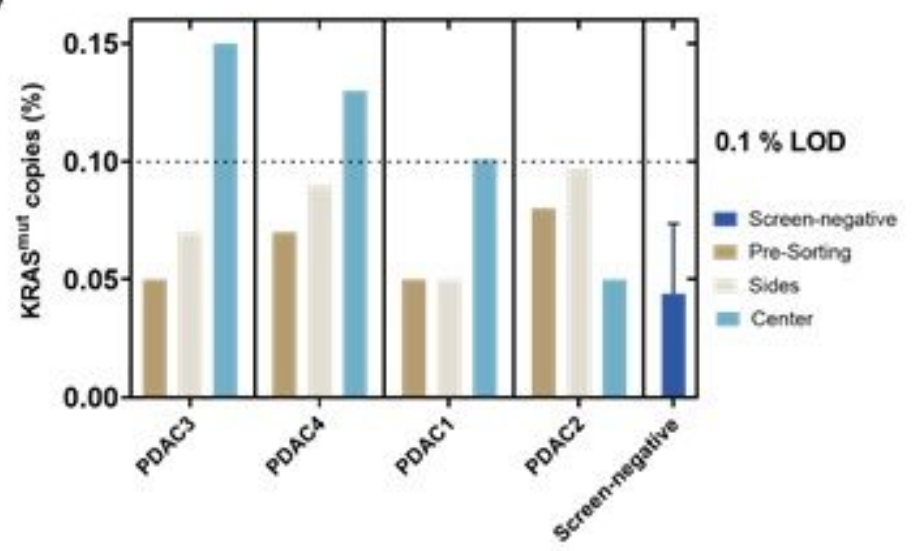

Figure 3

Downstream analysis of clinical PDAC samples processed with the DEP device. (A) In situ immunofluorescence microscopy analysis of PBMCs before device processing, arrows indicate presence of $\mathrm{CHCs}$ (Hoescht+/CD45+/CK+). (B) Average diameter $(\mu \mathrm{m})$ of $\mathrm{CHCs}$ and PBMCs from in situ immunofluorescence images. (C) Average clinical sample DNA extracted from cells collected from all outlets $(n=4)$. (D) Percent KRASmut copies for each patient $(n=4)$ from all outlets compared to average KRASmut copies for screen-negative controls $(n=5)$ measured by ddPCR.

\section{Supplementary Files}

This is a list of supplementary files associated with this preprint. Click to download. 
- nrreportingsummaryYC.pdf

- SupplementaryMaterial.pdf 